\title{
AVALIAÇÃO DA ISQUEMIA CEREBRAL FOCAL INDUZDA PELA OCLUSÃO DA ARTÉRIA CEREBRAL MÉDIA E A AÇÃO NEUROPROTETORA DO CETOPROFENO EM RATOS
}

\author{
LUIZANTONIO ARAUJ O DIAS*, BENEDICTO OSCAR COLLI**, \\ J OAQUIM COUTINHO NETTO*** , J OÃO J OSÉ LACHAT****
}

\begin{abstract}
RESUMO - A isquemia cerebral é fenômeno eventualmente observado durante procedimentos neurocirúrgicos e em patologias clínicas resultando em déficits neurológicos incapacitantes ou mesmo na morte. Por tratar-se de problema grave e de difícil solução, vários estudos têm sido efetuados com o objetivo de elucidar os mecanismos do fenômeno isquêmico no sistema nervoso central (SNC) e abolir ou diminuir seus efeitos através das drogas que protegem os neurônios (neuroprotetoras). Vários neurotransmissores estão envolvidos na isquemia e entre eles o glutamato destaca-se pela sua maior concentração no SNC. O objetivo deste estudo foi avaliar a isquemia cerebral focal em ratos através da dosagem do glutamato e dos achados morfológicos em uma evolução temporal e demonstrar uma possível ação neuroprotetora do cetoprofeno. Foram utilizados 36 ratos Wistar, subdivididos em 4 grupos: um grupo controle e outro sham; e outros dois em que os animais foram submetidos a isquemia pela oclusão seletiva da artéria cerebral média por um fio obstrutor durante 15, 30 e 45 minutos. Os animais de um destes grupos foram tratados com cetoprofeno 15 minutos antes da isquemia. A isquemia foi avaliada através de estudo histopatológico e da dosagem do glutamato extracelular in vitro. A análise morfológica mostrou não haver diferenças entre os animais normais e do grupo sham. Nos animais submetidos a isquemia, as alterações apareceram aos 30 minutos e acentuaram-se aos 45 . Os principais achados foram edema intersticial, desorganização cromatínica, vacuolização e desintegração nuclear. Os animais tratados com cetoprofeno apresentaram alterações semelhantes, porém menos intensas. Reduções nas dosagens in vitro do glutamato extracelular no córtex parietal dos animais submetidos a isquemia iniciaram-se a partir dos 30 minutos e acentuaram-se aos 45 e foram semelhantes nos animais com ou sem tratamento com cetoprofeno, indicando que esta droga parece não interferir com o metabolismo do glutamato na sinapse. Os achados histopatológicos no córtex parietal dos animais submetidos a isquemia , tratados ou não previamente com cetoprofeno, sugerem que esta droga tem um efeito neuroprotetor.
\end{abstract}

PALAVRAS-CHAVE: isquemia cerebral focal, rato, glutamato, histopatologia, neuroproteção, cetoprofeno.

\section{Focal cerebral ischaemia induced by midlle cerebral artery occlusion and the neuroprotective effect of} ketoprofen in rats

ABSTRACT - Cerebral ischaemia is eventualy observed during neurosurgical procedures and in several clinical entities that may cause severe neurological deficits and even death. Because it is a severe and complex problem, several studies have been done aiming to elucidate the mechanisms of the ischemic phenomenon and aiming to abolish or to diminish its effects, using drugs that protect the neurons from ischaemia-induced damage. Several neurotransmitters play a role in cerebral ischaemia with emphasis to glutamate by its high concentration in the central nervous system. The purpose of this study was to evaluate the effect of focal cerebral ischaemia in the rat through the dosage of the glutamate and morphological findings, and to evaluate a possible protective effect of the ketoprofen to ischemic neurons. Thirty-six rats Wistar were divided into four groups. The first was a control group,

Disciplina de Neurocirurgia do Departamento de Cirurgia do Hospital das Clínicas (HC) da Faculdade de Medicina de Ribeirão Preto (FMRP) da Universidade de São Paulo (USP): * Neurocirurgião, Chefe do Serviço de Neurocirurgia da Santa Casa de Ribeirão Preto; ** Professor Titular do Departamento de Cirurgia, Chefe da Disciplina de Neurocirurgia da FMRP/USP; *** Professor Associado do Departamento de Bioquímica da FMRP/ USP; **** Professor Doutor do Departamento de Morfologia da FMRP/USP.Aceite: 29-agosto-2000. 
the second a sham group and the animals of the third and fourth groups were submitted to induced cerebral ischaemia through selective obstruction of the midlle cerebral artery during 15, 30 and 45 minutes. Animals of the fourth group were previously treated with ketoprofen 15 minutes before the ischaemia. The ischaemia was evaluated through the histopathological examination and through dosage of the extracellular glutamate in vitro. The histopathological examination showed that there was no difference between the animals of the control and of the sham groups. In the animals submitted to ischemia histopathological alterations appeared at 30 minutes and become more intense at 45 minutes of ischaemia. The main findings were interstitial edema, chromatinic disorganization, vacuolization and nuclear desintegration. The animals treated with ketoprofen showed similar alterations, but they were less intense. Decrease in the dosage of glutamate in the parietal cortex of the animals submitted to ischaemia started at 30 minutes and became more intense at 45 minutes of ischaemia and was similar for animals previously treated or not with ketoprofen, indicating that this drug seems not to interfere with the metabolism of the glutamate at the synapses. The morphological findings in the parietal cortex of the animals submitted to ischaemia, previously treated or not with ketoprofen, suggest that this drug has a neuroprotective effect.

KEY WORDS: focal cerebral ischaemia, rat, glutamate, histopathology, neuroprotection, ketoprofen.

As lesões cerebrovasculares apresentam elevados índices de morbi-mortalidade, assim como os neurocirurgiões enfrentam situações semelhantes em procedimentos neurovasculares em que há necessidade de oclusão temporária de um vaso, principalmente nas cirurgia dos aneurismas cerebrais, com o objetivo de prevenir ou tratar hemorragias no campo operatório ${ }^{1}$. Estas podem levar a lesões hipóxico-isquêmicas de graus variados no sistema nervoso central (SNC), com repercussões clínicas, as quais podem ser graves para os pacientes ${ }^{2}$. $\mathrm{O}$ estudo dos fenômenos ligados a entidades clínicas que levam a hipóxia-isquemia do SNC são relevantes, pois visam a melhoria da qualidade de vida dos pacientes ${ }^{3}$. A alta demanda metabólica e a baixa reserva energética disponível rendem ao SNC susceptibilidade única frente às alterações na suplência de sangue, apesar da rede de circulação colateral muito desenvolvida neste. O SNC pode sofrer lesões isquêmicas globais e focais, e para ambas as situações existem modelos experimentais ${ }^{4}$. Não trataremos das lesões isquêmicas globais neste estudo. Nas lesões isquêmicas focais, existem três áreas distintas ${ }^{5}$ : (a) uma área central, onde o fluxo sanguíneo está acentuadamente diminuído; (b) uma próxima a esta, composta por tecido viável, embora com o fluxo diminuído, denominada área de penumbra; (c) uma área mais externa com características normais. Há grande número de neurotransmissores no SNC, sendo o glutamato o que se apresenta em maior quantidade e é o principal excitatório.

O glutamato está envolvido na síntese de proteínas e oligopeptídeos, na desintoxicação da amônia e na síntese do ácido fólico, e é precursor de outros neurotransmissores como o ácido gama amino butírico ${ }^{6}$. Ele é sintetizado no SNC a partir de três precursores (glutamina, glicose e ornitina), é armazenado nos terminais pré-sinápticos em vesículas e em forma livre, que são liberadas na fenda sináptica onde podem ser recaptadas, estimulam receptores pós sinápticos que permitem a entrada de cálcio para o interior das células, em especial os receptores do tipo NMDA (N-metil-Daspartato). Podem ser captadas pelas células gliais e sob ação das enzimas glutamina sintetase transformam-se em glutamina, a qual possui captação intensa e rápida nesses terminais e sob a ação da enzima glutaminase transforma-se em glutamina ${ }^{7}$. O glutamato participa na lesão neuronal isquêmica estimulando os receptores de canais de cálcio principalmente os do tipo NMDA ${ }^{8}$, permitindo a penetração intracelular de volumes exagerados de cálcio ${ }^{9}$, o qual nestas condições desencadeia eventos de cascatas enzimáticas ${ }^{10}$, que culminam com a morte neuronal; isto sumariza a teoria da toxicidade excitatória ${ }^{11}$. Existem vários modelos experimentais para se provocar isquemia focal, sendo a maioria deles por oclusão da artéria cerebral média $(\mathrm{ACM})^{12}$. Novas drogas às quais se atribuem propriedades ativas na proteção ou recuperação de neurônios isquemiados e ou anoxiados, encontram-se em diferentes estágios de avaliação, dentre eles o cetoprofeno ${ }^{10,13-15}$.

Os objetivos deste estudo foram, em ratos submetidos a isquemia cerebral focal por oclusão da ACM: 1. correlacionar a dosagem do glutamato com a evolução temporal do fenômeno isquêmico, 2. descrever a evolução temporal dos achados morfológicos do fenômeno isquêmico e 3. demonstrar a possível ação neuroprotetora do cetoprofeno ao fenômeno isquêmico. 


\section{MÉTODO}

Foram utilizados 36 ratos Wistar, com idade igual ou altamente aproximada (30 dias), porém sem possibilidade de possuirem doença aterosclerótica, com peso que variou de 280 a $320 \mathrm{~g}$, fornecidos pelo Biotério Central da FMRP-USP. Os animais foram divididos em quatro grupos: grupo 1, controle; grupo 2, sham; grupo 3 , subdividido em três subgrupos, os quais foram submetidos a isquemia de 15, 30 e 45 minutos cada, respectivamente; grupo 4, subdividido em três subgrupos com os mesmos tempos de isquemia, porém em todos foi injetado cetoprofeno previamente à isquemia. A realização da isquemia foi igual nos grupos 3 e 4 , seguindo Koiosumi et al. ${ }^{16}$, sendo os procedimentos realizados no Laboratório de Técnica Cirúrgica e Cirurgia Experimental do Departamento de Cirurgia, Ortopedia e Traumatologia da FMRP-USP.

Os animais foram anestesiados com halotano, entubados e conectados a um respirador para pequenos animais. A artéria ventral da cauda foi dissecada para controle da pressão arterial média (PAM), assim como a veia caudal ventral foi puncionada nos animais em que foi injetado cetoprofeno. Os parâmetros, como pressão endotraqueal, frequência respiratória, temperatura - mantida continuamente normal $\left(37-38^{\circ} \mathrm{C}\right) \mathrm{com}$ o auxílio de uma lâmpada de 220 watts - e PAM, foram mantidos dentro dos valores estabelecidos pela conexão com um biomonitor modelo (Hewlett Packard, USA). O fluxo de O2 foi mantido através de microfluxômetro de gases. Análise de gasometria arterial e glicose foram realizadas no setor de Bioquímica do mesmo laboratório e corrigidas conforme necessário. O procedimento cirúrgico iniciou-se por incisão mediana cervical, expondo-se o lado esquerdo com microdissecção auxiliada por microscópio cirúrgico (D. F. Vasconcelos) as artérias carótida comum esquerda (ACC), carótida interna (ACI) e carótida externa (ACE). A ACE é ligada, sendo o seu coto proximal utilizado para introdução do fio obstrutor, previamente confeccionado. Coloca-se um clipe tipo temporário (como utilizado nas cirurgias dos aneurismas cerebrais) na saída da artéria pterigopalatina, outro na ACC, introduzindo o fio obstrutor até imediatamente à saida da $\mathrm{ACM}$, obstruindo-a .O fio obstrutor e as medidas de sua introdução foram baseados em Koiosumi et al. ${ }^{16}$. Após os tempos de isquemia, assim como isquemia e injeção prévia de cetoprofeno na dose de $10 \mathrm{mg} / \mathrm{kg}$ em bolo nos animais do grupo 4 , os encéfalos foram retirados igualmente nos quatro grupos. Ambos os hemisférios eram mantidos irrigados com soro fisiológico gelado em uma placa de Petri mantida em recipiente com gelo picado. Os núcleos da base foram retirados. Na face externa dos hemisférios, com um punch oftalmológico de $7 \mathrm{~mm}$ de diâmetro, retirou- se um fragmento de cérebro, tendo como base a veia cerebral inferior e centro a ACM. O fragmento obtido do hemisfério esquerdo foi dividido em duas partes, uma parte para análise histopatológica e a outra para dosagem do glutamato. O mesmo procedimento foi realizado com o fragmento do hemisfério direito (lado especular). Os animais do grupo 1 (controle) foram submetidos à anestesia e estabilização hemodinâmica e gasométrica, após o que, foram retirados os encéfalos igualmente aos outros grupos. Nos animais do grupo 2 ( sham, traduzido do inglês como simulação) o procedimento foi realizado como nos grupos 3 e 4 , exceto que o fio obstrutor foi introduzido até certo ponto da ACI-E, sem obstrui-la.

As amostras de córtex cerebral foram processadas e analisadas no Laboratório de Neuroanatomia do Departamento de Morfologia da FMRP-USP como de rotina, pela inclusão em parafina e sendo realizados cortes de 6 micrômetros de espessura, corados com o tricrômico de Masson e analisados com um microscópio binocular Carl Zeiss. A documentação fotográfica foi feita com um fotomicroscópio Carl Zeiss (Axiophot I). As análises bioquímicas foram realizadas no Laboratório de Neuroquímica do Departamento de Bioquímica da FMRP-USP. Para análise dos aminoácidos contidos nos fragmentos de córtex cerebral, as amostras foram submetidas a cromatografia líquida de alto desempenho, em aparelho da marca Shimadzu, utilizando método descrito por Lindtroth \& Mopper ${ }^{17}$.

$\mathrm{Na}$ análise estatística foi utilizado o teste de Mann-Whitney para comparar amostras independentes e o teste de Kruskal Wallis, para comparar as dosagens do glutamato entre os diferentes tempos de isquemia nos grupos $3 \mathrm{e} 4$.

\section{RESULTADOS}

A análise histopatológica dos fragmentos dos cérebros dos animais dos grupos 1 e 2 não mostrou diferenças morfológicas no córtex dos animais dos grupos controle e sham (Figs 1 e 2). Entre os animais que não receberam infusão de cetoprofeno (grupo 3), nenhuma alteração foi observada naqueles submetidos a 15 minutos de isquemia. Nos animais submetidos a 30 minutos de isquemia, as alterações observadas foram edema intersticial focal, desorganização e fragmentação cromatínica, deslocamento do nucléolo e poucos núcleos desintegrados e vacuolizados (Fig 1), principalmente nas camadas 4,5 e $6^{18}$ do córtex cerebral. Naqueles submetidos a 45 minutos de isquemia, as lesões foram do mesmo tipo e nas mesmas camadas, porém mais intensas (Fig 1). Entre os animais que receberam a injeção prévia de cetoprofeno (grupo 4), nenhuma alteração foi observada 


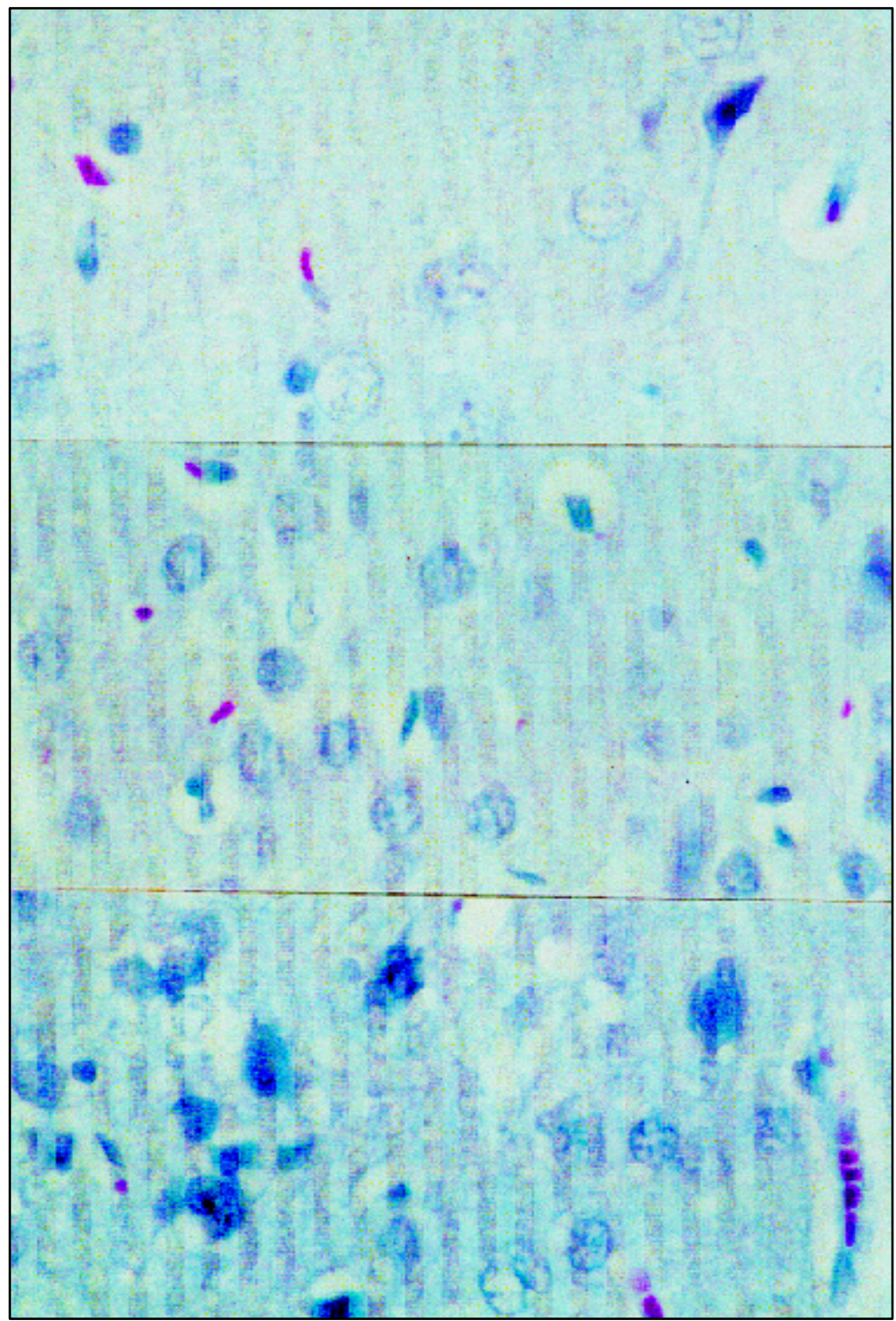

Fig 1. Fotomicrografias da camada 4 do córtex parietal esquerdo de rato Wistar (aumento: 125X. Coloração pelo Tricrômico de Masson). Em cima: animal do grupo 1 (normal). Centro: animal do grupo 3 submetido a 30 minutos de isquemia mostrando focos de edema intersticial leve, desorganização cromatínica e vacuolização nuclear. Em baixo: animal do grupo 3 submetido a 45 minutos de isquemia mostrando lesões semelhantes às anteriores, porém com maior intensidade.

naqueles submetidos a 15 minutos de isquemia e nos animais com 30 e 45 minutos de isquemia , os achados foram semelhantes aos do grupo 3, porém em menor intensidade (Fig 2).

Os valores médios da dosagem do glutamato de ambos os hemisférios nos grupos controle e sham são observados na Tabela 1, e não foram observadas diferenças significantes entre os níveis médios do glutamato em ambos os hemisférios. Nos animais submetidos a isquemia sem infusão de 


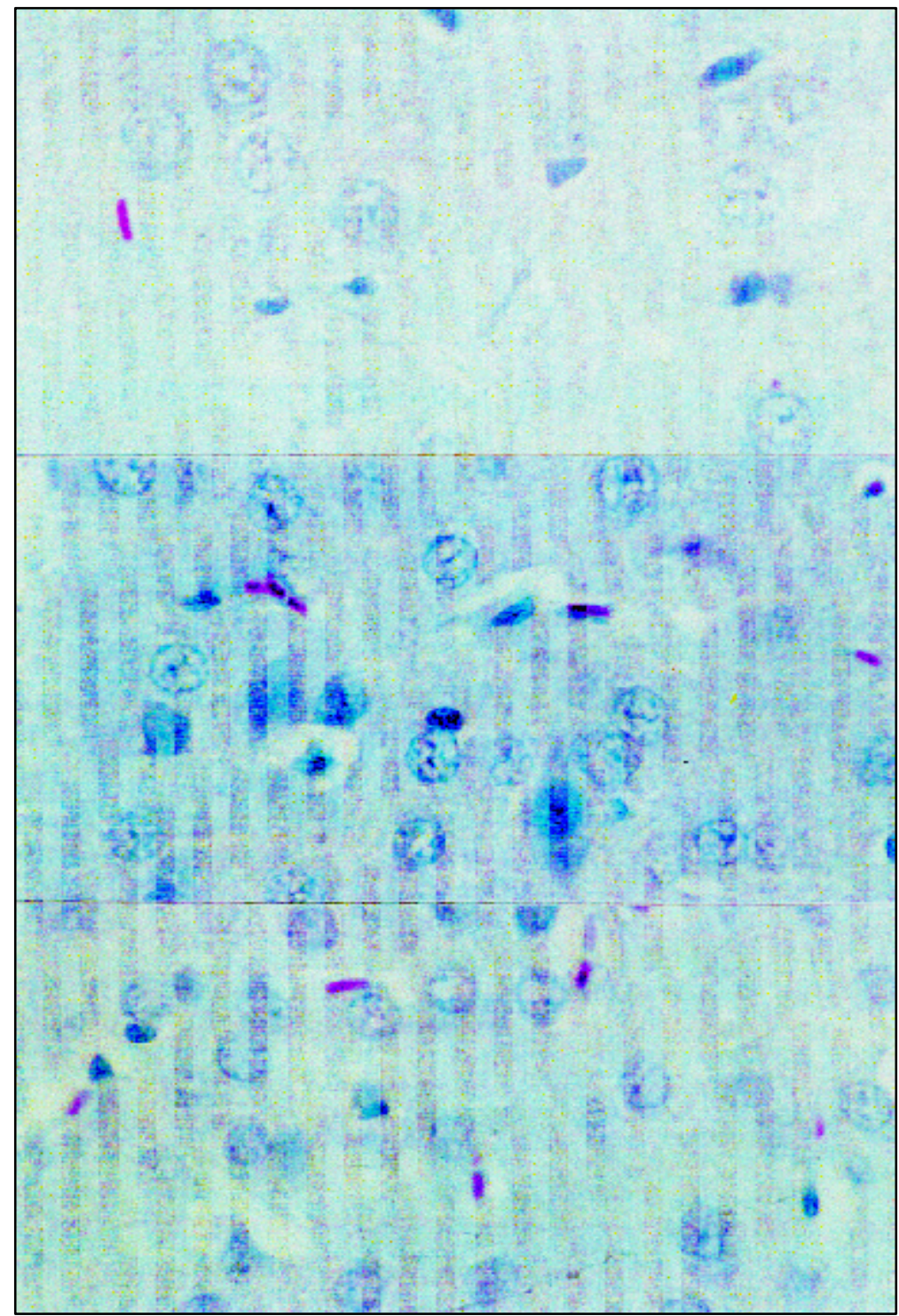

Fig 2. Fotomicrografia da camada 4 do córtex parietal esquerdo de rato Wistar (aumento: 125X. Coloração pelo Tricrômico de Masson). Em cima: animal do grupo 1 (normal). Centro e em baixo: animais do grupo 4 (tratados previamente com cetoprofeno) submetidos respectivamente a 30 e 45 minutos de isquemia mostrando lesões semelhantes aos correspondentes da Figura 1, porém com menor intensidade.

cetoprofeno (grupo 3), foi observada diminuição significativa entre os níveis médios de glutamato dos animais submetidos a 45 minutos de isquemia em relação àqueles submetidos a 30 minutos e estes apresentaram diminuição em relação aos submetidos a 15 minutos de isquemia $(45<30<15)$, para um nível de significancia de $\mathrm{p}=0,002$ (Tabela 2). Nos animais que receberam injeção prévia de cetoprofeno (grupo 4), os valores médios da dosagem de glutamato no córtex esquerdo daqueles submetidos a 45 minutos de isquemia foram menores que aos 30 e aos 15 minutos, porém os de 30 
Tabela 1. Valores médios da dosagem do glutamato no córtex parietal dos animais dos grupos controle e sham.

\begin{tabular}{lcc}
\hline Grupos de animais & $\begin{array}{c}\text { Dosagem de glutamato no hemisfério } \\
\text { esquerdo }(\mu \text { moles } / \mathrm{mg})\end{array}$ & $\begin{array}{c}\text { Dosagem de glutamato no hemisfério } \\
\text { direito }(\mu \text { moles } / \mathrm{mg})\end{array}$ \\
\hline Normais $(\mathrm{n}=3)$ & 0,19 & 0,18 \\
Sham $(\mathrm{n}=3)$ & 0,25 & 0,28 \\
\hline
\end{tabular}

Tabela 2. Valores médios da dosagem do glutamato no córtex parietal dos animais do grupo 3.

\begin{tabular}{lcc}
\hline Tempo de isquemia & $\begin{array}{c}\text { Dosagem de glutamato no hemisfério } \\
\text { esquerdo }(\mu \text { moles } / \mathrm{mg})\end{array}$ & $\begin{array}{c}\text { Dosagem de glutamato no hemisfério } \\
\text { direito }(\mu \mathrm{moles} / \mathrm{mg})\end{array}$ \\
\hline 15 minutos $(\mathrm{n}=5)$ & 0,24 & 0,28 \\
30 minutos $(\mathrm{n}=5)$ & 0,16 & 0,24 \\
45 minutos $(\mathrm{n}=5)$ & 0,09 & 0,19 \\
\hline
\end{tabular}

Tabela 3. Valores médios da dosagem do glutamato no córtex parietal dos animais do grupo 4.

\begin{tabular}{lcc}
\hline Tempo de isquemia & $\begin{array}{c}\text { Dosagem de glutamato no hemisfério } \\
\text { esquerdo }(\mu \text { moles } / \mathrm{mg})\end{array}$ & $\begin{array}{c}\text { Dosagem de glutamato no hemisfério } \\
\text { direito }(\mu \text { moles } / \mathrm{mg})\end{array}$ \\
\hline 15 minutos $(\mathrm{n}=5)$ & 0,23 & 0,26 \\
30 minutos $(\mathrm{n}=5)$ & 0,16 & 0,22 \\
45 minutos $(\mathrm{n}=5)$ & 0,11 & 0,20 \\
\hline
\end{tabular}

Tabela 4. Comparação entre os valores médios da dosagem do glutamato no córtex parietal esquerdo (isquemiado) dos animais dos grupos 3 e 4 .

\begin{tabular}{lcc}
\hline Tempo de isquemia & $\begin{array}{c}\text { Dosagem de glutamato no hemisfério esquerdo }(\mu \mathrm{moles} / \mathrm{mg}) \\
\text { Grupo } 3\end{array}$ & Grupo 4 \\
\hline 15 minutos $(\mathrm{n}=5)$ & 0,24 & 0,23 \\
30 minutos $(\mathrm{n}=5)$ & 0,16 & 0,16 \\
45 minutos $(\mathrm{n}=5)$ & 0,09 & 0,11 \\
\hline
\end{tabular}

não apresentaram diferenças em relação aos de 15 minutos $(45<30=15)$ para um nível de significância de $\mathrm{p}=0,01$ (Tabela 3). Comparando-se as dosagens médias do glutamato do hemisfério isquemiado, dos animais do grupo 3 e 4 , para cada um dos tempos de isquemia, não houve diferenças significantes (15 minutos: $\mathrm{p}=0,009 ; 30: \mathrm{p}=0,92$ e 45: $\mathrm{p}=0,14$ )(Tabela 4).

\section{DLSCUSSÃO}

A ocorrência e a extensão da isquemia, que ocorrem durante os procedimentos de clampagens arteriais temporárias e os déficits neurológicos subsequentes, dependem da duração da clampagem e da presença ou ausência de circulação colateral. Para que possamos atuar no processo evolutivo da isquemia cerebral, é fundamental o conhecimento dos eventos fisiopatológicos do fenômeno isquêmico, cuja sequência culmina com a morte e degeneração neuronal ${ }^{9,19,20}$. A constância do fluxo sanguíneo cerebral 
é importante para o fornecimento de glicose e $\mathrm{O} 2$ para o metabolismo aeróbico ${ }^{21,22}$. A redução de 25 a $40 \%$ do normal em uma zona de penumbra isquêmica é suportada pelo cérebro em torno de até 4 horas ${ }^{9}$. Reduções maiores levam a perda da homeostase iônica, que se inicia com a depleção de ATP, alterando os sistemas de transporte ativo, seguindo-se a abertura dos canais dos receptores tipo NMDA estimulados pelo glutamato, permitindo a penetração do $\mathrm{Ca}^{++}$em grande quantidade para o interior das células, o que desencadeia a cascata metabólica que culmina com a morte e lise celular ${ }^{11,23}$. O cetoprofeno é um antinflamatório não esteróide, neuroprotetor como um possível antagonista para receptor de glutamato tipo $\mathrm{NMDA}^{24}$, pela diminuição de receptores muscarínicos ${ }^{25}$.

$\mathrm{O}$ estudo visou à detecção das alterações precoces dos fenômenos isquêmicos, pela dosagem temporal do glutamato e mostrar sua neurotoxicidade em neurônios ${ }^{26,27}$, e a verificação das alterações histopatológicas precoces da isquemia e o eventual efeito neuroprotetor do cetoprofeno em um modelo experimental de isquemia por oclusão temporária da ACM em ratos. Este modelo mostrouse satisfatório, produzindo área isquemica relativamente constante, podendo ser usado em experimentos com reperfusão ${ }^{28}$. O período de isquemia utilizado, de até 45 minutos, foi adotado com o intuito de simular situações de clampagem temporária nos procedimentos neurovasculares, os quais geralmente não ultrapassam este período. A monitorização global do animal permitiu a manutenção da hemodinâmica em níveis comuns, impedindo os efeitos deletérios da hiper ou hipotensão arterial ${ }^{29}$, assim como da hipo ou hipertermia. A técnica cirúrgica é simples, porém exige passos técnicos rigorosos e treinamento prévio, sendo baseados no estudo de Carlotti ${ }^{28}$. O processo isquêmico foi provocado no hemisfério esquerdo, sendo o direito estudado como especular, baseandose no estudo de Morrell $^{30}$, em que se observou a propagação de atividade epiléptica de um foco primário para regiões distantes, induzindo nestas regiões epileptogênese. Porém, não observamos qualquer alteração importante na área contralateral à isquemia.

A análise morfológica qualitativa, pela inclusão em parafina e coloração pelo tricrômio de Masson, mostrou-se adequada por demonstrar os diferentes tipos celulares das diferentes camadas do córtex cerebral, como também os diferentes tipos e gravidade das lesões encontradas. Não realizamos análise morfológica quantitativa, por ser o período de isquemia utilizado de até 45 minutos, o qual é insuficiente para demonstrar degeneração e lise celular, o que é possível após esse período ${ }^{31}$. Nenhuma alteração foi observada em nenhum dos grupos aos 15 minutos de isquemia, pela abertura de colaterais alguns minutos após a queda na suplência do fluxo sanguíneo9 ${ }^{9}$ As lesões observadas aos 30 e 45 minutos com ou sem cetoprofeno, foram esparsas em focos pela área da lesão, em concordância com a literatura ${ }^{32}$.

A diminuição na dosagem do glutamato in vitro, observada em nosso estudo, sugere que o glutamato, ao passar para o meio extracelular, difunde-se rapidamente, é captado pelas células não isquêmicas, assim como é captado e transformado em glutamina pelas células da glia. Através da perfusão por microdiálise, in vivo, a dosagem do glutamato está aumentada naquele momento ${ }^{24}$.

Os achados histopatológicos sugerem uma ação neuroprotetora do cetoprofeno, porém, as dosagens do glutamato, mostram que o cetoprofeno parece não interferir com o metabolismo do glutamato na sinapse. $\mathrm{O}$ efeito neuroprotetor do cetoprofeno em nossas observações morfológicas não pode ser comprovado com a metodologia bioquímica por nós utilizada; porém, estudos da dosagem de $\mathrm{Ca}^{++}$intracelular, durante fenômenos isquêmicos em animais tratados e não tratados com cetoprofeno previamente, podem comprovar bioquimicamente a atividade neuroprotetora desta droga.

\section{Conclusões}

A metodologia empregada foi satisfatória para o estudo da isquemia focal permanente. Os achados histopatológicos tornaram-se evidentes a partir de 30 minutos, intensificando-se aos 45 . Os principais achados foram edema intersticial, desorganização cromatínica, vacuolização e desintegração nuclear. A queda das dosagens in vitro do glutamato extracelular iniciou-se a partir dos 30 minutos, acentuando-se aos 45 . Não se observaram diferenças significantes nas dosagens do glutamato nos 
animais que fizeram e que não fizeram uso de cetoprofeno, indicando que esta droga parece não interferir com o metabolismo do glutamato na sinapse. Os achados histopatológicos dos córtices isquemiados e tratados previamente com cetoprofeno foram parecidos com os dos córtices dos animais submetidos apenas a isquemia, porém em intensidade muito menor, sugerindo que esta droga tem um efeito neuroprotetor ao fenômeno isquêmico.

\section{REFERÊNCIAS}

1. Pool JL. Aneurysms of the anterior communicating artery, bifrontal craniotomy, and routine use of temporary clips. J Neurosurg 1961;18:98.

2. Zabramski JM, Hamilton MG. Cerebral vasospasm. In Spetzler RF, Carter LP (eds). Neurovascular surgery. New York: McGraw-Hill, 1994:583-601.

3. Terent A, Jonsson E. Costs of stroke in Sweden: a national perspective. Stroke 1994;25:2363-2369.

4. Colli BO, Nunes M, Carlotti CG Jr. Isquemia cerebral experimental. In Castro e Silva O, Beer A, Zuccoloto S (eds). Modelos experimentais de pesquisa em cirurgia. São Paulo: Probel Editora, 1998:643-662.

5. Pulsinelli W. Pathophysiology of the ischemic stroke. Lancet 1992;339:533-536.

6. Vanderberg CJ, Masom DF, Ronda G. A model of glutamate metabolism in brain: a biochemical analysis of a heterogenous structure. In Berl S, Clarke DD, Schneider D. Metabolic compartimentation and neurotransmission. Nato Adv Stud Inst Ser A Life Sci vol 6. New York: Plenum, 1975: 515-545.

7. Bradford HF, Ward H. Glutamine a major substract for serve endings. J Neurochem 1978;30:1453-1456.

8. Rothman SM, Olney JW. Excitotoxicity and the NMDA receptor. Trends Neurosci 1987;10:299-302.

9. Pulsinelli W. The ischemic penumbra in stroke. Scient Amer Med Sci 1995;2:16-25.

10. Lipton SA, Rosenberg PA. Excitatory amino acids as a final common pathway for neurologic disorders. N Engl J Med 1994;330:613-622.

11. Rothman SM, Olney JW. Glutamate and the pathophysiology of hipoxic-ischemic brain damage. Ann Neurol 1986;19:105-111.

12. Selman WR,Bhatti SU, Lust WD, Ratcheson .Pathophysiology of cerebral ischaemia . In Spetzler RF, Carter LP (eds). Neurovascular surgery. New York: McGra-Hill, 1994: 233-248.

13. Sandercock P, Willens H. Medical treatment of acute ischemic stroke. Lancet 1992;339:537-539.

14. Sucher NJ, Lei SZ, Lipton SA. Calcium channel antagonists attenuate NMDA receptor-mediated neurotoxicity in retinal ganglion cells in culture. Brain Res 1991;551:297-302.

15. Santos WA. Aminoácidos excitatórios na lesão neural seletiva por isquemia seguida de reperfusão: papel de antagonistas na proteção contra o processo isquêmico. Dissertação de Mestrado, Faculdade de Medicina de Ribeirão Preto. Ribeirão Preto, 1995.

16. Koiosumi J, Yoshida Y, Nakasawa T, Ooneda G. Experimental studies of ischemic brain edema: I. A new experimental model of cerebral embolism in rats in which recirculation can be introduced in the ischemic area. Jpn J Stroke 1986;8:1-8.

17. Lindroth $\mathrm{P}$, Mopper K. High performance liquid chromatography of subpicomole amounts of aminoacids by pre column fluorescence pre derivatization with o-phtaldeyde. Ann Chem 1979;51:1667-1677.

18. Zilles K, Wree A. Cortex: areal e laminar structures. In Paxinos G (ed). The rat nervous system. 2.Ed. Sydney: Academic Press, 1995: 649-685.

19. Siesjo BK. Pathophysiology and treatment of focal cerebral ischaemia: Part I. Pathophysiology. J Neurosurg 1992;77:169-184.

20. Zivin JA, Choi DW. Stroke therapy. Scient Amer 1991;265:36-43.

21. Ketz SS. The cerebral circulation. In Fishman AP, Richards DW. Circulation of the blood: men and ideas. New York: Oxford Univ Press, 1964:703-742.

22. Nakai H, Yamamoto YL., Diksic M, et al.. Triple-tracer autoradiograph demonstrate effects of hypoglycemia of rats. Stroke 1988;19:764-772.

23. Graham SH, Chen J. Limiting ischemic injury by inhibition of excitatory amino acid release. J Cer Blood Flow Metab 1993;13:88-97.

24. Santos WA. Aspectos bioquímicos e morfológicos da isquemia e reperfusão retiniana: estudo sobre a toxicidade excitatória, seus antagonistas e outros mediadores da lesão neuronal isquêmica. Tese de Doutorado, Faculdade de Medicina de Ribeirão Preto. Ribeirão Preto, 1999.

25. Asanuma M, Asanuma S, Gómez-Vargas M, Yamamoto M, Ogawa ND. Ketoprofen, a non-steroidal anti-inflamatory drug prevent the late onset of a muscarinic receptors in gerbils hippocampus after transient forebrain ischaemia. Neurosci Lett 1997;255:109-112.

26. Lucas F, Newhouse JP. The toxic effect of sodium L glutamate on the inner layers of the retina. Arch Ophthalmol 1957;58:193-204.

27. Olney JW. Glutamate-induced neuronal necrosis in the infant mouse hipotalamus: an electromicroscopy study. J Neuropathol Exp Neurol 1971;30:75-90.

28. Carlotti CG Jr. Estudo da respiração mitocondrial do córtex de ratos submetidos a isquemia focal pela oclusão da artéria cerebral média. Tese de Doutorado, Faculdade de Medicina de Ribeirão Preto. Ribeirão Preto, 1998.

29. Zhu CZ, Auer RN. Graded hypotension and MCA occlusion duration: effect in transient focal ischaemia. J Cer Blood Flow Metab 1995;15:980-988.

30. Morrell F. Secondary epileptogenic lesions. Epilepsia 1960;1:538-560.

31. Navarro IS, Pérez MPV, Silva MS, Gómez JMR, Sanz MV. Retinal ganglion cell death after different transient periods of pressure-induced ischaemia and survival intervals: a quantitative in vivo study. Invest Ophthalm Visual Sci 1996;37:2002-2014.

32. Altman J. Programmed cell death: the paths to suicide. Trends Neurosci 1992;15:278-280. 